\title{
Plant Growth Promoting Rhizobacteria as Biological Control Agent in Rice
}

\section{Duangkhaetita Kanjanasopa ${ }^{1 *}$, Wanthakarn Aiedhet ${ }^{1}$, Suraphon Thitithanakul ${ }^{1}$, Chanyarat Paungfoo-Lonhienne ${ }^{2}$}

\author{
${ }^{1}$ Agricultural Science and Technology Program, Faculty of Innovative Agriculture and Fishery Establishment Project, Prince of \\ Songkla University, Surat Thani Campus, Surat Thani, Thailand \\ ${ }^{2}$ School of Agriculture and Food Sciences, The University of Queensland, St Lucia, Queensland, Australia \\ Email: ^daungkhae.k@psu.ac.th, chanyarat@uq.edu.au
}

How to cite this paper: Kanjanasopa, D., Aiedhet, W., Thitithanakul, S. and Paungfoo-Lonhienne, C. (2021) Plant Growth Promoting Rhizobacteria as Biological Control Agent in Rice. Agricultural Sciences, 12, 1-8. https://doi.org/10.4236/as.2021.121001

Received: November 30, 2020

Accepted: January 8, 2021

Published: January 11, 2021

Copyright (๑) 2021 by author(s) and Scientific Research Publishing Inc. This work is licensed under the Creative Commons Attribution International License (CC BY 4.0).

http://creativecommons.org/licenses/by/4.0/

\begin{abstract}
The use of agrochemical products to combat diseases in crops has adverse effects on the environment and human health. Plant growth promoting rhizobacterium (PGPR) has been increasingly proposed as an eco-friendly alternative in agriculture. PGPRs have beneficial effects not only in promoting plant growth but also have shown their potential as biological control agent, being able to inhibit plant pathogens. Here, we investigated the use of PGPR Paraburkholderia sp. strain SOS3 to provide disease protection in rice (Oryza sativa L.). The antagonistic activity of SOS3 against five fungal pathogens of rice was assessed by dual culture on plates and on rice seedlings. The results showed that on plate assay, SOS3 inhibits the growth of Curvularia lunata, Rhizoctonia solani, Pyricularia oryzae, Helminthosporium oryzae, and Fusarium moniliforme by $17.2 \%, 1.1 \%, 8.3 \%, 32.5 \%$, and $35.4 \%$, respectively. When inoculated on rice seeds, SOS3 promotes seed germination and significantly reduces disease symptoms in plants infected with $R$. solani. These results suggest that SOS3 has a great potential to be used in rice agriculture to combat the "Sheath Blight" disease.
\end{abstract}

\section{Keywords}

Antifungal Activity, Biocontrol, Rice, Fungal Pathogen, Sustainable

Agriculture, Plant Growth-Promoting Rhizobacteria

\section{Introduction}

Protection of crop plants from pathogens and improvement of plant productivity is critical in the context of increasing demand for food to support the growing world population. Rice is an important crop worldwide, with over half of the 
world population depending on it for food [1]. Rice plants are attacked by various phytopathogens inducing diseases such as stem rot, blast, sheath blight, and bakanae, which result in low yield and quality of the crop [2] [3]. The application of agrochemicals remains the primary method to control microbial diseases. However, the use of synthetic chemicals has recently raised concerns due to their negative effects on the environment and human health, thus alternative and more sustainable strategies are required. Biological control is an environmentally friendly strategy for dealing with plant pathogens that shows potential for replacing or reducing the use of synthetic chemical compounds.

Plant growth promoting rhizobacteria (PGPR) are microbes associated with plant roots that promote plant growth by enhancing the availability of nutrients and minerals, and by producing plant hormones or other molecules that stimulate development. In addition, they are involved in inducing the resistance of the host plant to various biotic and abiotic stresses by releasing antimicrobial metabolites, synthesizing phytohormones, producing siderophores, and modulating the plant resistance responses [4]. Most of the PGPRs from the genus Bacillus, Pseudomonas, and Pantoea have been reported to be able to act as biological control agent (BCA) [5] [6] [7].

The SOS3 PGPR bacterium used in this study belongs to the Paraburkholderia genus. A recent study showed the positive effects of SOS3 in growth and nutrient acquisition in sugarcane [8]. Moreover, in Kikuyu grass (Pennisetum clandestinum), the bio-inoculation of SOS3 in soil increases the potency of organic fertlisers while reducing inorganic nitrogen leaching from soil to the environment [9]. Here we aimed to test if SOS3 is able to act as biocontrol agent and demonstrate that this PGPR has antagonist activities against rice fungal pathogens. Our results demonstrate that SOS3 protects rice against $R$. solani infection. Hence, it has the potential to be used as an effective biocontrol agent against the sheath blight disease.

\section{Materials and Methods}

\subsection{Plant Materials and Bacterial Strains}

Rice seeds (Oryza sativa L.) were derived from Chaiya fields at Surat Thani, Thailand. PGPR bacterium used in this study belongs to Paraburkholderia sp. strain SOS3 (Sustainable Organic Solution Pty Ltd.). It was cultivated at $30^{\circ} \mathrm{C}$ on Reasoner's 2A agar (R2A) medium. The pathogenic fungi, Curvularia lunata, Rhizoctonia solani, Pyricularia oryzae, Helminthosporium oryzae, and Fusarium moniliforme obtained from the Department of Agriculture, Ministry of Agriculture and Cooperatives, Thailand. The fungi were maintained at $4^{\circ} \mathrm{C}$ on potato dextrose agar (PDA) medium.

\subsection{Antifungal Activity}

The dual culture method was used to test the SOS3 antifungal activity against five fungal pathogens. Mycelial plugs of the 5-day-old culture of each fungal pa- 
thogen were placed in the center of PDA plate and incubated at $30^{\circ} \mathrm{C}$ for 3 days. Then, SOS3 grown on R2A agar plates was streaked straight line $2 \mathrm{~cm}$ at four sides of each agar disk at a distance of $3 \mathrm{~cm}$ from the mycelial plug, and the plates were incubated at $30^{\circ} \mathrm{C}$ for 7 days. Mycelial plugs grown on PDA plate without SOS3 were used as control. The experiment was done in four replications. The percentage of inhibition of radial growth (PIRG) was calculated by the following formula: \% PIRG $=\left(R_{1}-R_{2}\right) /\left(R_{1}\right) \times 100$, where $R_{1}$ is the radial distance growth of the fungal mycelia under control condition and $R_{2}$ is the radial distance of fungal mycelia growth in dual culture.

\subsection{SOS3 Antifungal Activity on Rice Seeds and Seedlings}

\section{Rice seed disinfection.}

Rice seeds were disinfected by treatment with $70 \%$ ethanol for 2 - 3 min, followed by surface sterilization with $15 \%$ and $10 \%(\mathrm{v} / \mathrm{v})$ chlorox $^{\oplus}$ with constant agitation for $15 \mathrm{~min}$ and $10 \mathrm{~min}$, respectively, and washed several times with sterile distilled water.

\section{Infection of rice seeds.}

SOS3 was grown to mid-log phase in nutrient broth medium (NB), centrifuged at $4500 \mathrm{x}$ gravity for $10 \mathrm{~min}$, washed twice, and re-suspended in $0.85 \% \mathrm{NaCl}$. The bacterial solution at an optical density of 1 (approximately $10^{9} \mathrm{cell} \mathrm{ml}^{-1}$ ) was used to soak disinfected rice seeds overnight. SOS3 treated seeds were transferred to a water suspension of each fungal pathogen $\left(10^{4}\right.$ $10^{6} \mathrm{cell} \mathrm{ml}^{-1}$ ) produced on PDA plates, along with bacteria-free control, for 2 hours.

\section{Growth of rice seedlings.}

Ten rice seeds of each treatment were grown into 1) bottles containing water agar [2\% (w/v) Phytagel ${ }^{\mathrm{TM}}$, Sigma Aldrich] 2) magenta box containing $20 \mathrm{~g}$ of sterilized potting mix prepared from peat:sand:perlite in a ratio 2:1:1. All treatments were set up in ten replicates and incubated at $25^{\circ} \mathrm{C} \pm 2{ }^{\circ} \mathrm{C}$ under 2000 3000 LUX illumination for a $16 \mathrm{~h}$ daily photoperiod. The percentage of seed germination, seedling infection by fungus, plant disease score, and plant dry weight were determined after an incubation of 14 days. Symptoms were scored as levels of infection using a susceptibility index of 0 , no evidence of disease; $1, \leq 10 \%$; $2, \leq 20 \% ; 3, \leq 50 \% ; 4, \leq 80 \%$; and $5, \leq 100 \%$ of leaf area affected (brown-black). To confirm the presence of the pathogens, $0.5 \times 0.5 \mathrm{~cm}$ squares of leaves presenting disease symptoms where stain with cotton blue dye. Stained hyphae were observed under a microscope at $400 \times$ magnification.

\subsection{Data Analysis}

Statistics were carried on using SPSS version 21.0. The data were analyzed using one-way analysis of variance (ANOVA) and differences between means were considered for $P<0.05$ by Duncan's multiple comparison (DMRT). 


\section{Results}

Five fungal pathogens were tested, Curvularia lunata, Rhizoctonia solani, Pyricularia oryzae, Helminthosporium oryzae, and Fusarium moniliforme, all being responsible for plant diseases in various crop plants in the world, such as sheath blight, blast, rot, and bakanae diseases [2] [3] [10] (Table 1). We first tested the potential antagonistic activity of SOS3 on agar plates (see Material and Methods), monitoring the direct effect that the PGPR has on the growth of the fungi. SOS3 considerably inhibited the growth of $F$. moniliforme, $H$. oryzae, and $C$. lunata (35\%,32\%, and $17 \%$, respectively) while it showed less effect against $R$. solani and P. oryzae (1\% and $8 \%$, respectively) (Figure 1 (a) and Table 1). We further investigated the antifungal property of SOS3 on rice seeds infected with each fungus. After 3 days of incubation on water agar plates, treatments with SOS3 showed a decrease of fungus growth on seeds infected by of $F$. moniliforme, H. oryzae, and C. lunata (Figure 1(b)), a result consistent with the experiments on agar plates.

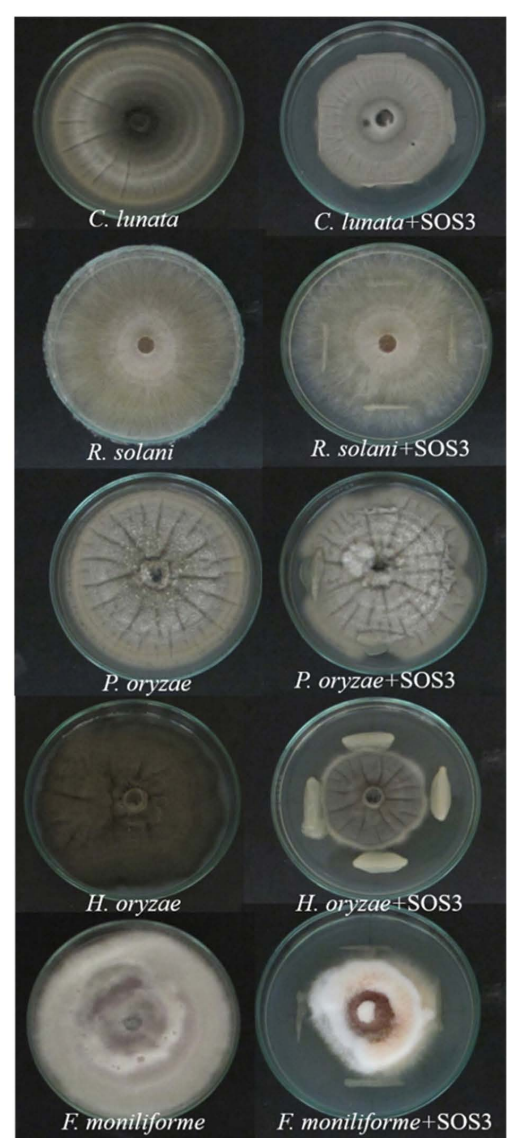

(a)

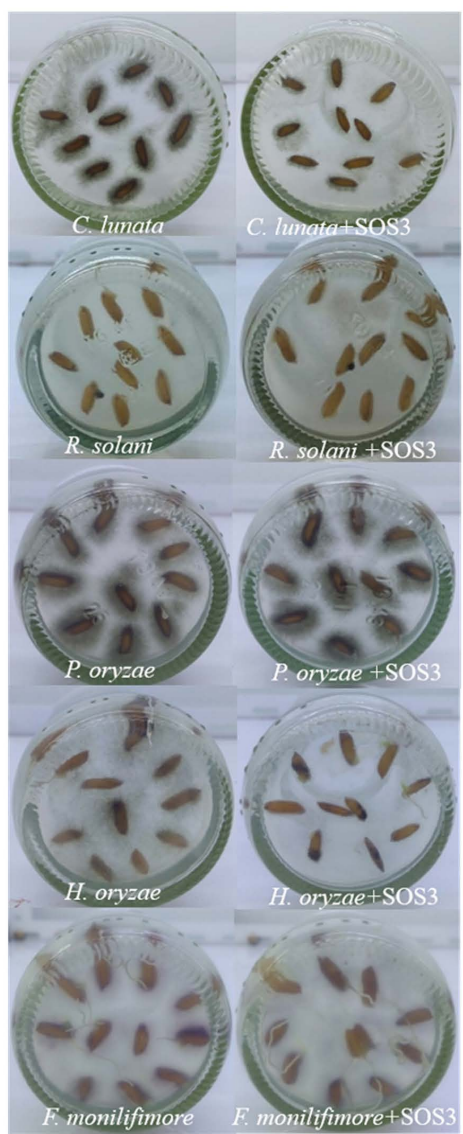

(b)

Figure 1. Antagonistic activity of SOS3 against five fungal pathogens. (a) The growth of the fungi was monitored on PDA plates in presence or absence (control) of SOS3. (b) Rice seeds inoculated by the fungi in presence or absence of SOS3 were incubated for three days. 
Table 1. Percentage inhibition of the growth on PDA plates of five fungal pathogens.

\begin{tabular}{|c|c|c|}
\hline Pathogens & Inhibition (\%) & Susceptible plants \\
\hline C. lunata & $17.18 \pm 7.71^{\mathrm{bc}}$ & Maize, Wheat, Soybean, Cucumber, Rice, Sugarcane, Millet \\
\hline R. solani & $1.08 \pm 1.08^{c}$ & Maize, Wheat, Soybean, Cotton, Peanut, Rice \\
\hline P. oryzae & $8.32 \pm 8.32^{c}$ & Wheat, Rice, Millet \\
\hline H. oryzae & $32.54 \pm 4.30^{\mathrm{ab}}$ & $\begin{array}{l}\text { Maize, Soybeans, Tomato, Sorghum, } \\
\text { Banana, Sugarcane, Peanut, Tobacco, Rice }\end{array}$ \\
\hline F. moniliforme & $35.41 \pm 1.93^{\mathrm{a}}$ & $\begin{array}{l}\text { Maize, Wheat, Soybeans, Tomato, } \\
\text { Sorghum, Cucumber, Cotton, Watermelon, Rice }\end{array}$ \\
\hline
\end{tabular}

Different lowercase alphabets indicate statistically significant differences between pathogen treatments at $P$ $<0.05$ (ANOVA, Duncan's post hoc test). Data represent averages and standard errors of four replicates.

The next step consisted of monitoring the effect of SOS3 on the germination of infected rice seeds and on the health of the corresponding seedlings. Rice was grown for 14 days on two different growth mediums. On one of the medium consisting of water agar plates, SOS3 had no effect on seeds germination. However, SOS3 reduced the percentage of infection of $P$. oryzae and the disease symptoms in plants infected by $R$. solani, P. oryzae, and $H$. oryzae. On the other medium consisting of magenta potting mix (see Material and Methods), the inoculation of seeds with SOS3 led to the increase of the percentage of germination of seeds infected by $R$. solani and to the decrease of the percentage of infection and disease symptoms of seedlings infected by the same fungus $(P<0.05$, Figure 3). The PGPR had no significant effects on plants infected with the other four pathogenic fungi. All together, the experiments on both mediums led to a common result showing that $\mathrm{SOS} 3$ has a prominent capacity to inhibit the pathogenicity of $R$. solani on rice.

It is remarkable that opposing results were obtained with the experiments related to inhibition of fungi growth by SOS3 (Figure 1) compared to those related to the effect of SOS3 on rice seedling infection (Figure 2 and Figure 3). For example, SOS3 shows strong antagonistic activity against both $C$. lunata and $F$. moniliforme on agar plates, however, it does not have significant effects on plants infected by those two pathogens. The reverse case happens when SOS3 is tested against $R$. solani, as SOS3 shows a great potential in rescuing plants infected with this pathogen although having almost no antagonistic activity against its growth on plate. This suggests that the metabolism of the pathogen and the PGPR are modified when they associate with the plant and highlight the importance of testing the potential biocontrol activity of a PGPR in real situation, when it is associated with host plants.

The main outcome of this research is that SOS3 appears to be a great candidate for the control of $R$. solani disease "sheath blight" in rice. $R$. solani is a great threat for rice, being responsible for important loss of grain yield and quality, which can represent up to $10 \%-50 \%$ yield loss in absence of protective action [11]. In China alone, the sheath blight costs 6 million tons of grains per year. 


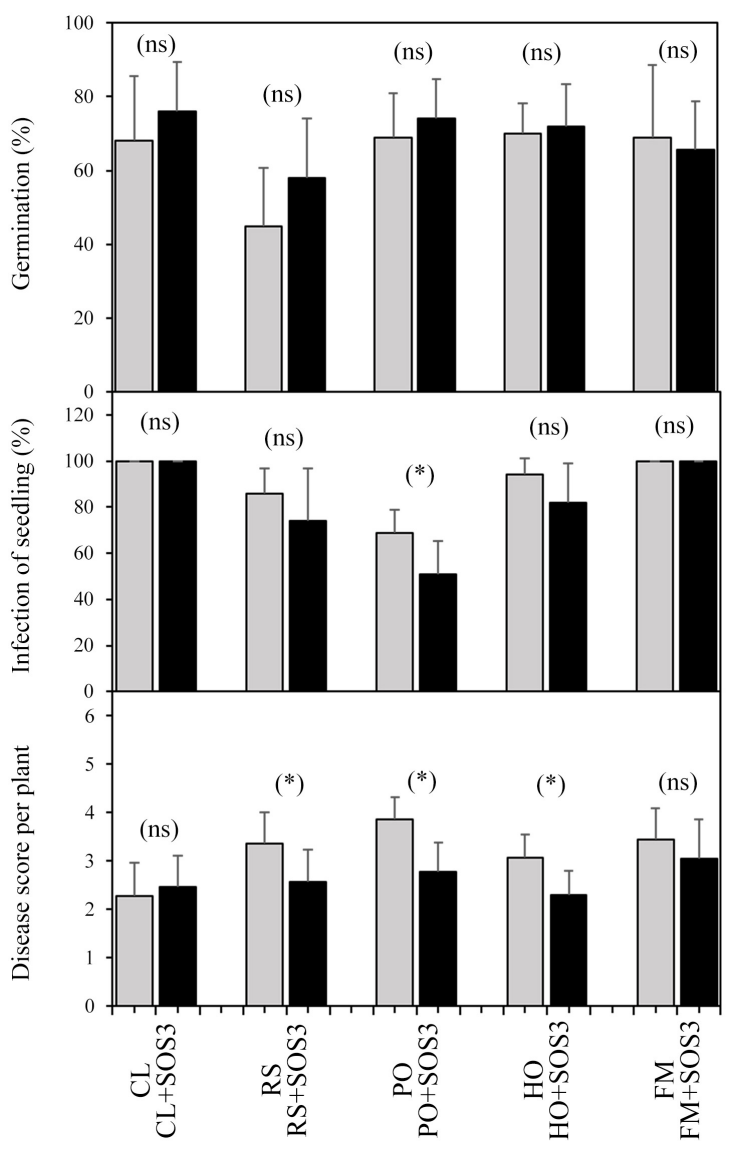

Figure 2. Biocontrol activity of SOS3 tested on rice infected by $C$. lunata, $R$. solani, $P$. oryzae, $H$. oryzae, and F. moniliforme, and grown on water agar for 14 days. The percentage of seed germination, seedling infection, and disease severity of seedling where monitored. Bars represent average with standard error of 10 replicates. Asterisks above bars indicate significant differences between treatment with SOS3 and control without SOS3 $(P<0.05)$.
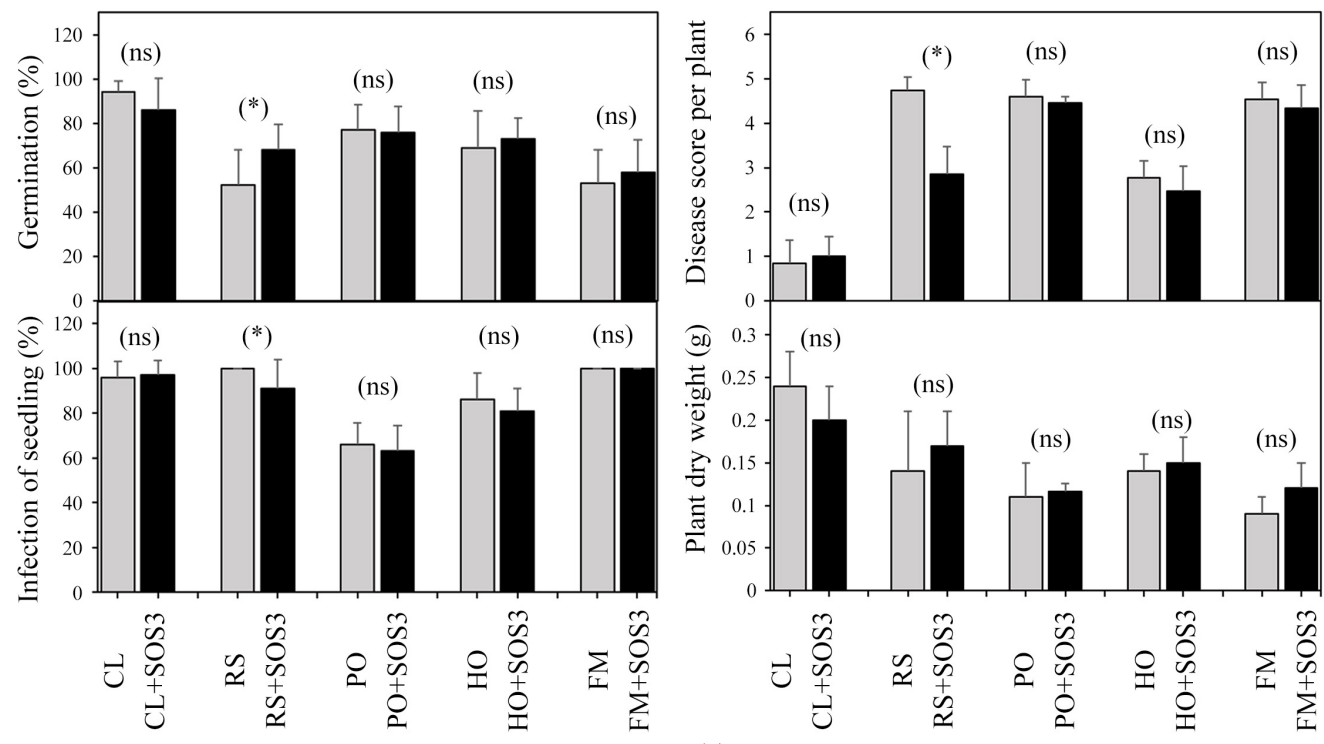

(a) 


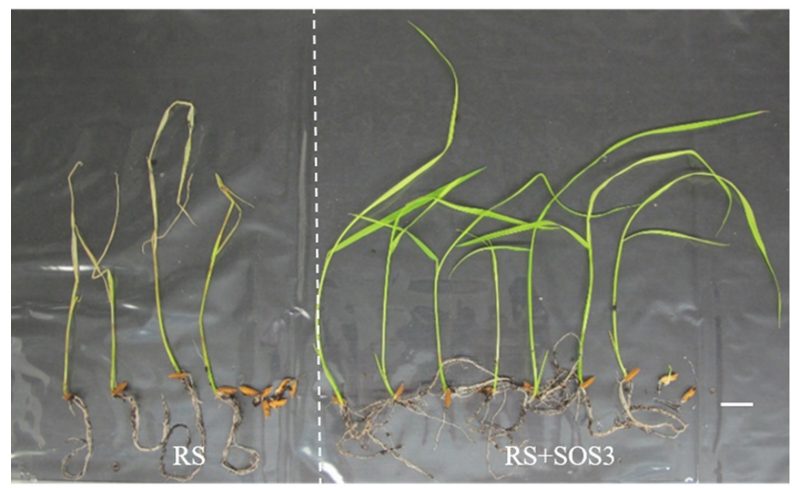

(b)

Figure 3. Biocontrol activity of SOS3 tested on rice infected by C. Iunata, R. solani, $P$. oryzae, $H$. oryzae, and F. moniliforme and grown on magenta potting mix for 14 days. (a) Percentage of seed germination, seedling infection, disease severity, and plant dry weight of infected seedlings. Bars represent average with standard error of 10 replicates. Asterisks above bars indicate significant differences between treatment with SOS3 and control without SOS3 $(P<0.05)$. (b) Pictures of rice seedlings harvested after 14 days, showing the beneficial effect that SOS3 has on plants infected by $R$. solani. Scale bar is $1 \mathrm{~cm}$.

In conclusion, SOS3 is a promising biocontrol agent in rice. Further research should evaluate its effect in field trials, and in parallele, the molecular mechanisms involved in the resistance against $R$. solani.

\section{Acknowledgements}

This research was funded by the Cooperative Research Project Grant

\section{CRCPFIVE000015.}

\section{Conflicts of Interest}

The authors declare that they have no known competing financial interests or personal relationships that could have appeared to influence the work reported in this paper.

\section{References}

[1] Khush, G. (2003) Productivity Improvements in Rice. Nutrition Reviews, 61, S114-S116. https://doi.org/10.1301/nr.2003.jun.S114-S116

[2] Saechow, S., Thammasittirong, A., Kittaakoop, P., Prachya, S. and Thammasittirong, S.N. (2018) Antagonistic Activity against Dirty Panicle Rice Fungal Pathogens and Plant Growth-Promoting Activity of Bacillus amyloliquefaciens BAS23. Journal of Microbiology and Biotechnology, 28, 1527-1535.

https://doi.org/10.4014/jmb.1804.04025

[3] Verma, S.K., Kingsley, K.L., Bergen M.S., Kowalski, K.P. and White, J.F. (2018) Fungal Disease Prevention in Seedlings of Rice (Oryza sativa) and Other Grasses by Growth-Promoting Seed-Associated Endophytic Bacteria from Invasive Phragmites australis. Microorganisms, 6, 21. https://doi.org/10.3390/microorganisms6010021

[4] Rosier, A., Medeiros, F.H. and Bais, H.P. (2018) Defining Plant Growth Promoting Rhizobacteria Molecular and Biochemical Networks in Beneficial Plant-Microbe 
Interactions. Plant and Soil, 428, 35-55. https://doi.org/10.1007/s11104-018-3679-5

[5] Glick, B.R. (2012) Plant Growth-Promoting Bacteria: Mechanisms and Applications. Scientifica, 2012, Article ID: 963401. https://doi.org/10.6064/2012/963401

[6] Khan, N., Martínez-Hidalgo, P., Ice, T.A., Maymon, M., Humm, E.A., Nejat, N., Sanders, E.R., Kaplan, D. and Hirsch, A.M. (2018) Antifungal Activity of Bacillus Species against Fusarium and Analysis of the Potential Mechanisms Used in Biocontrol. Frontiers in Microbiology, 9, 2363.

https://doi.org/10.3389/fmicb.2018.02363

[7] Jiang, L., Jeong, J.C., Lee, J., Park, J.M., Yang, J., Lee, M.H., Choi, S.H., Kim, C.Y., Kim, D., Kim, S.W. and Lee, J. (2019) Potential of Pantoea dispersa as an Effective Biocontrol Agent for Black Rot in Sweet Potato. Scientific Reports, 9, Article No. 16354. https://doi.org/10.1038/s41598-019-52804-3

[8] Paungfoo-Lonhienne, C., Watanarojanaporn, N. and Jaemsaeng, R. (2020) Plant Growth Promoting Rhizobacteria Enhance the Efficiency of the Combination of Organic and Chemical Fertilisers in Sugarcane. Open Journal of Ecology, 10, 440-444. https://doi.org/10.4236/oje.2020.107028

[9] Paungfoo-Lonhienne, C., Redding, M., Pratt, C. and Wang, W. (2019) Plant Growth Promoting Rhizobacteria Increase the Efficiency of Fertilisers While Reducing Nitrogen Loss. Journal of Environmental Management, 233, 337-341. https://doi.org/10.1016/j.jenvman.2018.12.052

[10] Awla, H.K., Kadir, J., Othman, R., Rashid, T.S., Hamid, S. and Wong, M.Y. (2017) Plant Growth-Promoting Abilities and Biocontrol Efficacy of Streptomyces sp. UPMRS4 against Pyricularia oryzae. Biological Control, 112, 55-63. https://doi.org/10.1016/j.biocontrol.2017.05.011

[11] Yellareddygari, S.K.R., Reddy, M.S., Kloepper, J.W., Lawrence, K.S. and Fadamiro, H. (2014) Rice Sheath Blight: A Review of Disease and Pathogen Management Approaches. Journal of Plant Pathology and Microbiology, 5, 241.

$\underline{\text { http://doi.org/10.4172/2157-7471.1000241 }}$ 